\title{
Reinitiating Warfarin: Relationships Between Dose and Selected Patient, Clinical and Hospital Measures
}

\author{
Lucas G. Leonhard; Richard L. Berg, MS; James K. Burmester, PhD; \\ Joseph J. Mazza, MD; John R. Schmelzer, PhD; and Steven H. Yale, MD
}

\begin{abstract}
Background: Warfarin is an oral anticoagulant used in the long-term treatment/prevention of venothromboembolic disease. Patients undergoing elective surgical and non-surgical procedures may require temporary warfarin discontinuation followed by reinitiation after their procedure. Because little information is available regarding best methods for warfarin reinitiation, we investigated current practices to inform management decisions.
\end{abstract}

Methods: Subjects were required to have a known and stable warfarin dose prior to discontinuation, which was operationalized by requiring, within 7-days prior to discontinuation, that they have at least one INR in therapeutic range (2.0-3.5), no INR(s) out of range, and no more than a $15 \%$ change in warfarin dose. Stable dose prior to discontinuation was defined as the average daily dose received in the 7 days immediately prior to discontinuation. Reinitiation dose was defined as the average daily dose received in the first 3 days after warfarin was restarted. Subjects were divided into three groups based on whether they received approximately the same, a higher, or a lower dose at reinitiation and were also grouped by calendar time into three distinct periods that reflected differing levels of availability of electronic and patient care data that may impact reinitiation dose decisions. These groupings facilitated analyses and descriptions of trends in reinitiation dosing and supported other analyses, including tests for association between dose group and selected subject demographic, clinical, medication and hospitalization measures. All study data were abstracted from Marshfield Clinic electronic patient care and administrative databases and electronic patient care databases from Ministry St. Joseph's Hospital (Marshfield,WI).

Results: We identified 205 subjects with warfarin temporarily discontinued between 1994 and 2012: 99 subjects in same dose group, 32 subjects in the low group, and 74 subjects in the high group. Because relatively wide differences were observed in the proportion of same dose subjects during more recent years (2007-20I2) compared to earlier years (54\% vs $35 \%$ ), we focused our analyses on this recent period, which included 140 subjects. Review of physician notes and other documents yielded virtually no information about reasons for reinitiation dose decisions. In addition, tests for association between reinitiation dose group and subject demographic, clinical, medication and hospital measures were uniformly uninformative.

Conclusions: We observed varied dosing strategies for reinitiating patients on warfarin and, in more recent years, an apparent trend toward reinitiating patients on the same dose. However we could not associate dosing strategy with specific patient demographic, clinical, medication or hospital factors. Many factors influence whether a physician reinitiates a patient at a different dose than his/her prior stable warfarin dose. However, in the absence of clinical indications for modification, we believe patients with a previously established effective dose should be reinitiated at that same dose following temporary warfarin discontinuation.

Keywords: Anticoagulants/administration \& dosage; Anticoagulants/adverse effects;

Anticoagulants/therapeutic use; Warfarin/administration, dosage, therapeutic use

Corresponding Author: Steven H. Yale, MD; North Florida Regional Healthcare; 6500 Newberry Road; Gainesville, FL 32605; Tel: (352) 313-8258; Email: steven.yale@hcahealthcare.com

\author{
Received: October II, 2013 \\ Revised: February 20, 2014 \\ Accepted: March 14, 2014 \\ doi: $10.3121 / \mathrm{cmr} .2014 .1208$
}

Financial Support: This study was supported in part by the Herman and Gwendolyn Shapiro Summer Research Program, University of Wisconsin School of Medicine and Public Health; and in part through philanthropic support of the Marshfield Clinic Research Foundation Summer Student Research Internship Program. 
W management for treatment and prevention of thromboembolic events. ${ }^{1,2}$ Patients may require temporary discontinuation of warfarin before undergoing surgical and non-surgical procedures to allow their international normalization ratio (INR) to return to baseline to decrease the risk of bleeding..$^{3-5}$ Following the medical procedure or period of increased bleeding risk, patients requiring long-term therapy may be restarted on a standard institutional dose, their previous dose, or a higher or lower dose, based on clinical judgment.

During the past 15 years, many institutions, including Marshfield Clinic, have developed a separate outpatient department whose sole purpose is to manage patients' anticoagulation. At the Marshfield Clinic, this department is called the Anticoagulation Service (ACS), also known as the "Coumadin Clinic." Patients can go to the ACS Monday through Saturday to have their blood drawn, INR measured, and warfarin dose adjusted if needed. However when a patient is hospitalized his or her anticoagulation management becomes temporarily the responsibility of a physician, most typically a general internist, specialist, or hospitalist.

Despite vast literature on warfarin initiation and management, to our knowledge, optimal strategies for reinitiation after temporary discontinuation of warfarin has not been developed or standardized across medical institutions. ${ }^{3,5}$ Only one case series with 36 patients attempted to answer this question, using time to therapeutic INR as the primary outcome. ${ }^{6}$ Strategies for warfarin reinitiation are not discussed in the American College of Physician guideline for warfarin managment. ${ }^{8}$ Given the limited study results available, we undertook this retrospective, observational study to investigate dosing patterns during warfarin reinitiation and their impacts on clinical measures normally assessed in warfarin management. Because our study subjects had been previously stabilized on a warfarin dose, we were especially interested in examining and characterizing relationships between reinitiation dose and prior stable dose, examining trends in reinitiation dose decisions that might result from advances in electronic health records and physician support tools, and through review of physician notes and other data, exploring if there were common patient characteristics and/or conditions that were systematically associated with reinitiation dose.

\section{Methods}

Following institutional review board approval with waiver of informed consent, subjects were identified from the combined electronic medical record of Marshfield Clinic, Ministry St. Joseph's Hospital, St. Clare's Hospital, and Flambeau Hospital in Wisconsin during the period from January 1994 to August 2012. All other study data were available from Marshfield Clinic's electronic health record, Marshfield Clinic/St. Joseph's Hospital (Marshfield) combined medical record, and other administrative data sources.
Individuals were required to have a known and stable warfarin dose to be eligible for the study. This was operationalized by requiring that all subjects, within 7-days prior to discontinuation, have at least one INR in range, no INR(s) out of range, and no more than a $15 \%$ change in warfarin dose. An INR $<2$ was also required for the $1^{\text {st }}$ INR following reinitiation to ensure adequate withdrawal of warfarin prior to reinitiation. These requirements selected for patients on long-term warfarin therapy whose regimens did not greatly fluctuate prior to discontinuation. Subjects were excluded if they received fresh frozen plasma (FFP) or vitamin K during warfarin discontinuation, if they discontinued warfarin but were not reinitiated, if they were not on a stable warfarin dose before discontinuation, or if there was inadequate information available to determine warfarin dose. Hospital and clinic records were used to abstract dates of warfarin discontinuation and reinitiation, and dose of warfarin received daily for 2 weeks before discontinuation and 4 weeks following reinitiation.

Stable dose prior to discontinuation and reinitiation dose were determined to support comparisons between them and to other subject characteristics and clinical factors. Stable dose prior to discontinuation was defined as the average daily dose received in the 7 days immediately prior to discontinuation. Reinitiation dose was defined as the average daily dose received in the first 3 days after dosing was restarted.

Subjects were classified into one of three mutually exclusive groups (same, high, low) based on the relationship between their reinitiation dose and their prior stable dose. When the reinitiation dose differed from prior stable dose by $15 \%$ or less (absolute value), subjects were deemed to be on the same dose. Else, subjects were classified into a high group when reinitiation dose was higher than prior stable dose by more than $15 \%$ or into a low group when reinitiation dose was more than $15 \%$ less than prior stable dose.

We used this reinitiation dose classification to begin our examination of reinitiation dose decisions over time, expecting to observe generally increasing proportions of reinitiation dose in the same group as we moved forward in time. Because improvements in electronic medical records, supporting databases, and practice innovations (e.g., ACS) developed during the study period, we investigated shifts in reinitiation dosing over time.

Based on our institutional advancements in care processes, we chose three periods that corresponded to years 1994-2000, 2001-2006 and 2007-2012, respectively. The 1994-2000 period preceded the establishment of our anticoagulation service. The 2001-2006 period corresponds to the establishment and early development of the anticoagulation service. The final period (2007-2012) corresponds to a time frame with a fully mature anticoagulation service and an enhanced data warehouse facility that should have made prior warfarin dose data more readily available for reinitiation decisions. 
Table 1. Summary of reinitiation dose classification by study period.

\begin{tabular}{lcccc}
\hline & N & $\begin{array}{c}\text { Low } \\
\mathbf{n}(\%)\end{array}$ & $\begin{array}{c}\text { Same } \\
\mathbf{n}(\%)\end{array}$ & $\begin{array}{c}\text { High } \\
\mathbf{n}(\%)\end{array}$ \\
\hline $1994-2000$ & 13 & $3(23 \%)$ & $3(23 \%)$ & $7(54 \%)$ \\
$2001-2006$ & 52 & $9(17 \%)$ & $20(38 \%)$ & $23(44 \%)$ \\
$2007-2012$ & 140 & $20(14 \%)$ & $76(54 \%)$ & $44(31 \%)$ \\
Total & 205 & $32(16 \%)$ & $99(48 \%)$ & $74(36 \%)$ \\
\hline
\end{tabular}

We reviewed and abstracted physician and hospital progress notes during the time period immediately prior to and following reinitiation dose. We were very interested in understanding physician reinitiation dose decisions generally, and especially when reinitiation dose was markedly different than prior stable dose. There can be many important reasons for such differences; our interests were simply to document and categorize them to the extent possible.

We abstracted select demographic and clinical data for each subject, which included selected comorbid conditions, severity of illness, medication exposures, and in-patient factors. We did not attempt to capture all possible data within these broad categories because it was beyond the scope of this relatively small, observational study. Additionally, we did not believe we would have adequate statistical power to support multiple analyses of these data. Instead, we sought to characterize the patterns of reinitiation dose with these patient and hospital factors for descriptive purposes only.

\section{Results}

Data were available to classify 205 study subjects into one of the three reinitiation dose groups; 99 subjects (48.3\%) were classified into the same group, 74 subjects (36.1\%) into the high group, and 32 subjects $(15.6 \%)$ into the low group (table 1). In more recent years (2007-2012), slightly over
$54 \%$ of subjects were reinitiated on same dose compared to $35 \%$ in study years prior to 2007 . Based on the clustering of our study data across multi-year periods, considering advancements in recent years in systems and processes that support anticoagulation management, and because more recent periods are likely more indicative of current clinical management practices, we focused our remaining analyses on reinitiations during 2007-2012.

Mean and median prior dose and reinitiation dose by reinitiation dose group are reported in Table 2. These data are helpful for understanding the range in dose within and across reinitiation dose groups. Clearly, there is variability in reinitiation dose decisions. It appears there is some regression toward the common warfarin dosing of about $4.5-5 \mathrm{mg} /$ day.

Maximum attained INR within up to 28 days of reinitiation was highest in the high group (8.0) and virtually identical in the low group (6.4) and same group (6.3). The percentage of subjects with at least one INR $>3.5$ varied only slightly among reinitiation dose groups, ranging from 30.8\% (low group) to $32.3 \%$ (high group) (table 2).

We were unable to document with any detail or consistency physician decision criteria related to reinitiation dose decisions. Consequently, we are unable to report results from these efforts.

While we anticipated that documentation of such decisions would be inconsistent, the dearth of related information and documentation was unanticipated, especially in a clinical care setting with extensive electronic medical record capabilities that span both inpatient and outpatient environments.

We investigated potential associations among reinitiation dose group and selected subject demographic, comorbid

Table 2. Summary of stable dose and reinitiation dose data and selected clinical measures by reinitiation dose group, 2007-2012.

\begin{tabular}{|c|c|c|c|c|}
\hline & Low & Same & High & Total \\
\hline \multicolumn{5}{|l|}{ Prior Daily Dose (mg) } \\
\hline Mean & 5.6 & 5.1 & 3.6 & 4.7 \\
\hline Median & 5.1 & 4.6 & 3.3 & 4.3 \\
\hline Minimum & 1.6 & 1.6 & 1.1 & 1.1 \\
\hline Maximum & 11.1 & 15.0 & 10 & 15 \\
\hline \multicolumn{5}{|l|}{ Reinitiation Daily Dose (mg) } \\
\hline Mean & 3.6 & 5.0 & 5.5 & 5.0 \\
\hline Median & 3.3 & 4.8 & 5.0 & 4.7 \\
\hline Minimum & 1.3 & 1.7 & 2.2 & 1.3 \\
\hline Maximum & 7.5 & 15.0 & 18.3 & 18.3 \\
\hline Maximum INR* & 6.4 & 6.3 & 8.0 & \\
\hline$\%$ of patients with INR $>3.5^{\star}$ & $30.8 \%$ & $31.5 \%$ & $32.3 \%$ & \\
\hline
\end{tabular}

Prior dose $=$ average daily dose for 7 days prior to discontinuation

Reinitiated dose $=$ average daily dose for first 3 days of re-initiation

*INR data reflect from 3 to 28 days of follow-up. 
Table 3. Results of tests for association between reinitiation dose group and selected continuous demographic, patient condition, and treatment-related factors, 2007-2012.

\begin{tabular}{|c|c|c|c|c|c|c|c|c|c|c|}
\hline \multirow[b]{2}{*}{ Variable } & \multicolumn{3}{|c|}{ Low } & \multicolumn{3}{|c|}{ Same } & \multicolumn{3}{|c|}{ High } & \multirow{2}{*}{$\begin{array}{c}P \\
\text { value }\end{array}$} \\
\hline & $\mathbf{N}$ & Mean & Median & $\mathbf{N}$ & Mean & Median & $\mathbf{N}$ & Mean & Median & \\
\hline $\begin{array}{l}\text { Age at } \\
\text { reinitiation } \\
\text { (years) }\end{array}$ & 20 & 79.5 & 81.3 & 76 & 75.6 & 78.5 & 44 & 75.2 & 76.4 & ns \\
\hline $\begin{array}{l}\text { Days } \\
\text { hospitalized } \\
\text { (days) }\end{array}$ & 20 & 10.7 & 8.0 & 74 & 6.3 & 6.0 & 44 & 7.5 & 6.5 & ns \\
\hline $\begin{array}{l}\text { Charlson } \\
\text { index } \\
\text { (value) }\end{array}$ & 20 & 3.7 & 4.0 & 76 & 3.0 & 3.0 & 44 & 3.2 & 3.0 & ns \\
\hline $\begin{array}{l}\text { Restart to } \\
\text { ACS follow- } \\
\text { up (days) }\end{array}$ & 19 & 8.3 & 6.0 & 76 & 6.1 & 6.0 & 39 & 5.8 & 5.0 & ns \\
\hline $\begin{array}{l}\text { eGFR } \\
\text { (value) } \\
\text { Serum }\end{array}$ & 20 & 53.3 & 53.2 & 76 & 57.9 & 56.3 & 44 & 56.9 & 51.6 & ns \\
\hline $\begin{array}{l}\text { albumin } \\
\text { (value) }\end{array}$ & 20 & 3.6 & 3.8 & 76 & 3.7 & 3.7 & 44 & 3.7 & 3.8 & ns \\
\hline
\end{tabular}

conditions, reasons for discontinuing warfarin, severity of illness, medication exposures, and in-patient factors, primarily for descriptive purposes. Results from these investigations are summarized in table 3 (continuous measures), table 4 (categorical measures) and table 5 (medication exposures). None of these potential associations demonstrated a consistent, reliable relationship with reinitiation dose group.

\section{Discussion}

Although warfarin is the most commonly prescribed drug for the treatment and prevention of thrombotic events, there remains considerable uncertainty regarding the best methods for its reinitiation when the drug needs to be temporarily discontinued. ${ }^{10-12}$ Here, we investigated and described warfarin reinitiation dosing patterns; investigated the bases for clinical decisions regarding reinitiation dose; and described relationships among dosing patterns, patientspecific conditions, and clinical factors.

Our investigation included development of data to support descriptive analyses of warfarin reinitiation and qualitative reviews of subject medical record data, including physician notes, to determine documentation that supported physician decisions regarding reinitiation dose. Our three classification grouping of prior stable and reinitiation dose relationships indicated that over the entire 19-year study period slightly more than $48 \%$ of subjects were reinitiated on a warfarin dose that was the "same" as their prior stable dose (ie,

Table 4. Summary of tests for associations between reinitiation dose group and selected categorical demographic, co-morbid conditions, and treatment-related factors, 2007-2012).

\begin{tabular}{|c|c|c|c|c|}
\hline & Low (\%) & Same (\%) & High (\%) & Fisher's $P$ \\
\hline Male & 50.0 & 50.0 & 52.3 & ns \\
\hline Hypertension & 80.0 & 72.4 & 88.6 & ns \\
\hline Active malignancy & 10.0 & 5.3 & 6.8 & ns \\
\hline Diabetes & 45.0 & 32.9 & 31.8 & ns \\
\hline Heparin bridging & 20.0 & 23.7 & 29.5 & ns \\
\hline Reinitiation on Mon-Wed & 50.0 & 64.5 & 70.5 & ns \\
\hline Discontinued for: & & & & $\mathrm{ns}^{*}$ \\
\hline Coronary Catherization & 25.0 & 32.9 & 27.3 & \\
\hline Endoscopy & 10.0 & 5.3 & 6.8 & \\
\hline Surgery & 40.0 & 23.7 & 31.8 & \\
\hline Other & 25.0 & 38.2 & 34.1 & \\
\hline
\end{tabular}

*Test for association across all reasons for warfarin discontinuation 
Table 5: Summary of tests for associations between reinitiation dose group and medication exposures, 2007-2012.

\begin{tabular}{lcccc}
\hline & Low (\%) & Same (\%) & High (\%) & Fisher's $\boldsymbol{P}$ \\
\hline Amiodarone Group & 15.0 & 3.9 & 4.5 & $\mathrm{~ns}$ \\
Any statin & 35.0 & 56.6 & 56.8 & $\mathrm{~ns}$ \\
Atorvastatin & 5.0 & 6.6 & 3.8 & $\mathrm{~ns}$ \\
Simvastatin & 25.0 & 35.5 & 29.5 & $\mathrm{~ns}$ \\
Cephalosporin antibiotics & 25.0 & 26.3 & 11.4 & $\mathrm{~ns}$ \\
Antiplatelet drugs & 10.0 & 11.8 & 11.4 & $\mathrm{~ns}$ \\
Clopidogrel & 5.0 & 10.5 & & $\mathrm{~ns}$ \\
\hline
\end{tabular}

ns, not significant

differed by no more than $\pm 15 \%)$. In more recent years (20072012), slightly over $54 \%$ of subjects were reinitiated on same dose compared to $35 \%$ in study years prior to 2007 .

While there are no other studies against which to benchmark these results, our impression is that the approximate $54 \%$ rate of same dose reinitiation toward study end reflects not only real valuable advances in clinical management over time but also opportunities for continued improvements. Afterall, considerable time, effort and skill in dose adjustment is often required to obtain a stable warfarin dose for a patient. Once a stable dose has been established for a patient why would a physician reinitiating warfarin ignore such important information, especially when the drug is discontinued only temporarily to accommodate a procedure.

Our analyses of demographics, clinical conditions, and medication exposures yielded no insights into any systematic relationships with reinitiation dose decisions. There were no systematic relationships evident among dosing groups and demographic factors, measures of patient condition severity, comorbidities and procedures, use of bridging therapies or discharge dispositions to nursing homes or rehabilitation facilities. There were also no systematic relationships between reinitiation dose and administration of statins (any), or atorvastatin or simvastatin, cephalosporin antibiotics, antiplatelate drugs, and (even) amiodarone group drugs, which have a well-documented effect on warfarin dose.

We find interesting the general lack of relationships with antibiotics in particular, and drugs overall. Our previous work in warfarin initiation ${ }^{13}$ which was conducted during the latter part of this study period, revealed that early initiation dose adjustments were often made in anticipation of an INR response to an inpatient's exposure to a new antibiotic therapy. However study results indicate a more random pattern among the three dose groups. We would have expected a more systematic pattern where reinitiation dose would be generally lower (higher) than prior stable dose, depending on the expected INR-related effect of the antibiotic.

Our review of medical record data documenting physician rationales for specific reinitiation dosing decisions yielded almost no information regarding why a specific reinitiation dose was chosen. While we anticipated that documentation of such decisions would be inconsistent, the dearth of related information and documentation was unanticipated. Consequently, the content richness associated with physician decision processes related to reinitiation dose decisions could not be ascertained. Among the issues we hoped to explore were decisions to reinitiate patients on higher (lower) doses when their prior stable doses were much lower (higher) than the customary $5 \mathrm{mg} /$ day. The patterns in our data are suggestive of a mean reversion-type dosing process where the mean is unspecified but is between $5 \mathrm{mg} /$ day and the patient's prior stable dose whenever the latter is more than $\sim 25 \%$ different from $5 \mathrm{mg} /$ day. However, unless physician reinitiation dosing decisions can be more fully documented, our observations should be considered as conjecture.

Although our study was largely exploratory in nature, we did record bleeding and bruising events during data abstraction even though we knew we would not have statistical power to analyze them in a formal manner. For descriptive purposes only, we note that we observed a total of 26 minor bleeding or bruising events during the study period; and no serious or fatal bleeding events. The distribution of the minor bleeding and bruising events among the three dosing groups was uninformative.

Our investigation of warfarin reinitiation dosing also yielded qualitative findings that may be valuable in planning future investigations. Major limiting factors in the study were the availability and timing of INRs. These factors impacted the number of subjects eligible for study by limiting our ability to establish a stable prior warfarin dose for comparative purposes. They also limited our analyses options for assessing INR response to reinitiation dose decisions. These limiting factors are directly related to the retrospective nature of the study. A well-designed prospective study, perhaps a pragmatic clinical trial, which standardizes the timing of both prediscontinuation and reinitiation INRs, would provide more uniform INR data to enhance analyses. It could also strengthen our understanding of physician decision-making related to reinitiation dose by collecting such data systematically in real time, thereby avoiding the significant data limitations we 
encountered. Finally, such an undertaking would support analyses of important patient and clinical outcomes, such as serious bleeding and thrombotic events, which were beyond the scope of our investigation.

Until more data, including important physician decisionrelated data on dose, are developed, important questions related to best clinical practices for reinitiating individuals on warfarin will remain unanswered.

\section{Acknowledgement}

The authors thank Marshfield Clinic Research Foundation's Office of Scientific Writing and Publication for editorial assistance in the preparation of this report.

\section{References}

1. Glurich I, Burmester JK, Caldwell MD. Understanding the pharmacogenetic approach to warfarin dosing. Heart Fail Rev 2010;15:239-248.

2. Horton JD, Bushwick BM. Warfarin therapy: evolving strategies in anticoagulation. Am Fam Physician 1999;59:635-646.

3. Palaniswamy C, Selvaraj DR. Periprocedural bridging anticoagulation: current perspectives. Am J Ther 2011;18:e89-e94.

4. du Breuil AL, Umland EM. Outpatient management of anticoagulation therapy. Am Fam Physician 2007; 75:1031-1042.

5. Broderick JP, Bonomo JB, Kissela BM, Khoury JC, Moowaw CJ, Alwell K, Woo D, Flaherty ML, Khatri P, Adeoye O, Ferioli S, Kleindorfer DO. Withdrawal of antithrombotic agents and its impact on ischemic stroke occurrence. Stroke 2011;42:2509-2514.

6. Schultz KT, Bungard TJ. Dosing Options for decreasing the time to achieve therapeutic anticoagulation when reinitiating warfarin: a case series. Pharmacotherapy 2011;31:793805.

7. White RH, McKittrick T, Hutchinson R, Twitchell J. Temporary discontinuation of warfarin therapy: changes in the international normalized ratio. Ann Intern Med 1995; $122: 40-42$.

8. Guyatt GH, Akl EA, Crowther M, Schunemann HJ, Gutterman DD, Zelman Lewis S; American College of Chest Physicians. Introduction to the ninth edition: Antithrombotic Therapy and Prevention of Thrombosis, 9th ed: American College of Chest Physicians Evidence-Based Clinical Practice Guidelines. Chest 2012;141:48S-52S.

9. Tomisti L, Del Re M, Bartalena L, Tanda ML, Pucci A, Pambianco F, Bogazzi F. Effects of amiodarone, thyroid hormones and CYP2C9 and VKORC1 polymorphisms on warfarin metabolism: a review of the literature. Endocr Pract 2013;19:1043-1049.

10. Kearon C. Management of anticoagulation in patients who require invasive procedures. Semin Vasc Med 2003; 3:285-294.

11. Jaffer AK, Brotman DJ, Chukwumerije N. When patients on warfarin need surgery. Cleve Clin J Med 2003;70:973-984.

12. Hylek EM, Regan S, Go AS, Hughes RA, Singer DE, Skates SJ. Clinical predictors of prolonged delay in return of the international normalized ratio to within the therapeutic range after excessive anticoagulation with warfarin. Ann Intern Med 2001;135:393-400.

13. Burmester JK, Berg RL, Yale SH, Rottscheit CM, Glurich IE, Schmelzer JR, Caldwell MD. A randomized controlled trial of genotype-based coumadin initiation. Genet Med 2011;13:501-518.

\section{Author Affiliations}

Lucas G. Leonhard",'; Richard L. Berg, MS*;

James K. Burmester, PhD,', Joseph J. Mazza, MD*;

John R. Schmelzer, PhD*; Steven H. Yale, MD ${ }^{*, 2}$

${ }^{*}$ Clinical Research Center, Marshfield Clinic Research Foundation, Marshfield, WI, USA.

'University of Wisconsin, Madison, WI USA.

*iomedical Informatics Research Center, Marshfield Clinic

Research Foundation, Marshfield, WI, USA.

${ }^{1}$ Current affiliation: Gundersen Health System, La Crosse, Wisconsin USA

${ }^{2}$ Current affiliation: North Florida Regional Healthcare, Gainesville, Florida USA 